\title{
Endogenous levels of reducing sugars, free amino acids and phenols during various stages of in vitro culture of cotton (Gossypium Spp.)
}

\author{
Olawole O. OBEMBE ${ }^{1 *}$, Tanveer KHAN ${ }^{2}$, Jacob O. POPOOLA ${ }^{1}$, \\ Anil K. SINGH ${ }^{2}$ and R.C. PANT ${ }^{2}$
}

\author{
${ }^{I}$ Applied Biology and Biotechnology Unit, Department of Biological Sciences, College of Science and \\ Technology, Covenant University, PMB 1023 Ota, Ogun State, Nigeria. \\ ${ }^{2}$ Department of Plant Physiology, CBSH, GBPUA\&T, Pantnagar,Uttaranchal-India-263145 \\ "Corresponding author; E-mail: odun_wole@yahoo.co.uk; Tel : +2348130928965
}

\begin{abstract}
Somatic embryogenesis is widely preferred as the regeneration route for in vitro studies in cotton. However, the regeneration efficiency through this approach is low; a problem that is believed to be as a result of the biochemical properties of the plant. The objective of this study was to investigate possible relationships between three biochemical factors (reducing sugars, phenols, and free amino acids) and somatic embryogenesis. In vitro cultures of the different embryogenic and non-embryogenic cultivals were established. The levels of reducing sugars, phenols and free amino acids were determined at different developmental stages of the cultures. Higher levels of reducing sugars and lower level of phenol were observed in embryogenic cultivars compared to their non-embryogenic counterparts. There was a general increase in the levels of free amino acids, which decreased with time in the highly embryogenic cultivars, whereas the levels remained high in the poorly embryogenic and non-embryogenic cultivars. The higher content of phenols and free amino acids may be implicated in the poor somatic embryogenic response. The data show that there are factors that may serve as markers of somatic embryogenesis in cotton, which need to be empirically determined for any particular cultivar chosen for genetic improvement through embryogenesis.
\end{abstract}

(C) 2010 International Formulae Group. All rights reserved.

Keywords: Somatic embryos, biochemical studies, embryogenic and non-embryogenic, somatic embryogenesis, biomarkers.

\section{INTRODUCTION}

Cotton (Gossypium spp.) is credited to be one of the most ancient and important commercial crops next only to the food grains and occupies the prime position among the cultivated fiber crops in India. It is a global crop with a raw material value of $\$ 5.5$ billion. The world cotton area and production is 32.4 million ha and 87.4 million bales, respectively. Cotton has been considered as recalcitrant for in vitro culture. Regeneration has been reported in some cotton cultivars through somatic embryogenesis (Sakhanokho et al., 2001; Kumria et al., 2003; Khan et al., 2006). The preference for somatic embryogenesis as a regeneration route is hinged on the single cell origin of the somatic embryos (Merkle et al., 1995). A prolonged 
culture period, high frequency of abnormal embryo development, low conversion rate of somatic embryos into plantlets, and lack of shoot elongation are the problems associated with cotton regeneration. Thus, a proper understanding of the factors affecting somatic embryogenesis and recalcitrance in cotton is a prerequisite for the development of reproducible regeneration protocol, which is critical for the tissue culture-based genetic improvement, may further pave a way for the development of genetically modified plants.

Somatic embryogenesis (SE) is influenced by a number of different endogenous factors, as well as exogenously applied phytohormones, that affect various biochemical pathways leading to morphogenesis. Some of these factors have been widely studied in other crops such as carrot, rice, cocoa and eucalyptus (Huang and Liu, 2002; Alemannoh et al., 2003; Pinto et al., 2008) but very few of such studies are known in cotton.

The levels of sugars for example, govern cellular differentiation during in vitro culture. It has been speculated that different sugars, especially hexoses and sucrose and intermediates of sugar metabolism may have a signaling function, in morphogenesis (Iraqi and Tremblay, 2001). Also, phenol accumulation has been implicated in playing some roles in morphogenesis, even though there are divided opinions as to whether or not it influences morphogenesis negatively. Cotton, being a woody species has the problem of phenol exudation, which is a major hurdle in the establishment of a stable cell culture. Somatic embryogenesis is a stress related phenomenon (Kumria et al., 2003), as such high levels of endogenous free amino acids (FAA) in the plant is generally regarded as stress indicators.

The main objective of the study was to elucidate the mechanism involved in morphogenesis and embryogenesis of cotton with respect to establishing the correlations between embryogenesis and endogenous levels of reducing sugars, free amino acids and phenol in the embryogenic (BD-1, BD-6, Sarvottam, Jawahar Tapti, Coker 312 FR, SH131 and LH-900) and non-embryogenic cultivars (JK-4 and Khandwa-2) at different stages of development. It should be noted that SH-131 and LH-900 are poorly embryogenic while others are highly embryogenic (Khan et al., 2006). Coker 312 FR, a highly embryogenic cultivar was taken as a standard. Additionally, Coker 312 FR, SH-131, LH900, Khandwa-2, JK-4 are tetraploid $G$. hirsutum cultivars while BD-1, BD-6, Sarvottam and Jawahar Tapti are diploid $G$. arboretum.

\section{MATERIALS AND METHODS}

The seeds of different cultivars were washed in running tap water and then with sterile distilled water. The seeds were thereafter treated in step-wise manner, with $70 \%$ ethanol for $5 \mathrm{~min}$., sodium hypochlorite (4\% available chlorine) for $10 \mathrm{~min}$ and $0.1 \%$ $(\mathrm{w} / \mathrm{v})$ mercuric chloride for $15 \mathrm{~min}$. After each sterilization treatment, seeds were rinsed three times with sterilized distilled water. The seeds were then soaked for 5-6 hrs in sterilized distilled water to soften the seed coat. For germination, the seed coat was removed with forceps. The seeds were placed aseptically on $0.5 \%$ agar solidified medium, pH-5.8, containing MS (Murashige and Skoog, 1962) inorganic salts, vitamins and sucrose for germination. Initially, the cultures were maintained in dark for $24 \mathrm{hrs}$ and then incubated for two days at $27 \pm 1{ }^{\circ} \mathrm{C}$ under cool white fluorescent light with an intensity of 40$60 \mathrm{~mol} \mathrm{~s}^{-1} \mathrm{~m}^{-2}$ with a photoperiod of $16 \mathrm{hrs}$. Cotyledonary leaf explants from 3 days old seedlings were used to induce callus on MS medium supplemented with $9.04 \mu \mathrm{M}$ dichlorophenoxy acetic acid and $0.464 \mu \mathrm{M}$ kinetin while different combinations of phytohormones were used to induce callus on hypocotyl explants (Table 1). After 20 days, the callus was transferred to MS medium supplemented with $0.452 \mu \mathrm{M} \quad 2,4-$ 
dichlorophenoxy acetic acid (2,4-D), for proliferation. Levels of reducing sugars, phenols and free amino acids were determined as follows:

\section{Determination of reducing sugars levels}

The levels of the reducing sugars were determined by dinitrosalicylic method according to Sadasivam and Manickam (1996). $5 \mathrm{ml}$ of $80 \%$ ethanol was added to 100 mg of callus crushed to a paste and then kept on a boiling water-bath to evaporate ethanol. The residue was dissolved in $5 \mathrm{ml}$ of distilled water (also used for FAA and phenol). $2 \mathrm{ml}$ of DNS reagent was added to $1 \mathrm{ml}$ of the dissolved ethanol extract and heated in boiling water-bath for $5 \mathrm{~min}$. $1 \mathrm{ml}$ of tartarate reagent (40\% potassium sodium tartarate) was then added when the content was still warm. A deep red colour formation indicates the presence of reducing sugars. The intensity was measured at $575 \mathrm{~nm}$.

\section{Determination of total free amino acids}

The method for the determination of the free amino acids was carried out according to Sadasivam and Manickam (1996) as given below. $1 \mathrm{ml}$ of the dissolved ethanol extract, above, was taken and added to equal volume of ninhydrin solution (prepared by dissolving $0.8 \mathrm{~g}$ of $\mathrm{SnCl}_{2} 2 \mathrm{H}_{2} \mathrm{O}$ in $500 \mathrm{ml}$ of $0.2 \mathrm{M}$ Citrate buffer $\mathrm{pH}-5.0$ and then adding it to 500 $\mathrm{ml}$ of methyl cellosolve i.e 2- methoxyethanol ). The absorbance was recorded after $15 \mathrm{~min}$ at $570 \mathrm{~nm}$. The amount of amino acids in the sample was determined by plotting the absorbance against the standard curve of glycine (20-100 $\mu \mathrm{g})$ after 20, 40 and 60 days of culture, which correspond to the callus induction, proliferation and embryo induction stages, respectively.

\section{Determination of total phenol}

$1 \mathrm{ml}$ of folin ciocalteau's reagent and $80 \%$ ethanol were mixed in the ratio 1:1. 0.5 $1.0 \mathrm{~g}$ of callus tissue was weighed and crushed in a mortar pestle and 10 volumes of $80 \%$ ethanol was added. The homogenate was centrifuged at 10,000 rpm for $20 \mathrm{~min}$. The pellet was allowed to dry, and redissolved in $5 \mathrm{ml}$ of distilled water. $50 \mu \mathrm{l}$ of the extract was taken and made up the volume to $3 \mathrm{ml}$ with distilled water. $0.5 \mathrm{ml}$ of Folin Ciocalteau's reagent and $2 \mathrm{ml}$ of $20 \% \mathrm{Na}_{2} \mathrm{CO}_{3}$ were then added step-wise to the extract. This was then mixed thoroughly and boil for $1 \mathrm{~min}$. The absorbance was then taken at $650 \mathrm{~nm}$, after cooling.

\section{Statistical analysis}

All the experiments were performed thrice with three replicates in each case. The means were compared at the $\mathrm{p}=0.05$ level of significance and analyzed by ANOVA using Tukey's HSD.

\section{RESULTS \\ Callus induction}

Hypocotyl and cotyledonary leaf explants from in vitro-raised seedlings of different $G$. arboreum and $G$. hirsutum cultivars were cultured on MS medium supplemented with various combinations and concentrations of phytohormones. (Table 1). Cotyledonary leaves of all the cultivars formed callus on MS medium supplemented with $9.04 \mu \mathrm{M} 2,4-\mathrm{D}+0.464 \mu \mathrm{M}$ kinetin (Kin). Hypocotyl explants of $G$. arboreum cultivars formed callus when cultured on a medium containing $10.74 \mu \mathrm{M}$ Naphthalene acetic acid (NAA) $+4.64 \mu \mathrm{M}$ (Kin) while those of $G$. hirsutum cultivars formed callus on MS medium supplemented with 5.37 $\mu \mathrm{M}$ NAA + $0.464 \mu \mathrm{M}$ Kin and $14.9 \mu \mathrm{M}$ 2-isopentenyl adenine $(2 \mathrm{iP})+0.537 \mu \mathrm{M}$ NAA. Callus was induced from both explants within a week and well-developed callus was formed within 20 days.

The rate of callus induction and proliferation was faster and higher in $G$. arboreum cultivars than $G$. hirsutum cultivars, with the exception of Coker 312 FR, in both 
explants. After 20 days, the callus was transferred to MS medium supplemented with $0.452 \mu \mathrm{M} 2,4-\mathrm{D}$, for proliferation.

\section{Induction of somatic embryogenesis}

The proliferated callus was then transferred to the embryogenic medium (Table 1). Cultivars BD-1, BD-6, Sarvottam and Jawahar Tapti were very similar in their response (Figure 1). MS medium supplemented with $0.452 \mu \mathrm{M} 2,4-\mathrm{D}$ was found to be the best for induction of somatic embryogenesis. The embryogenic callus of $G$. arboreum cultivars gave rise to welldeveloped somatic embryos, when transferred to MS medium containing $0.68 \mu \mathrm{M} 2,4-\mathrm{D}+$ $2.98 \mu \mathrm{M}$ 2-isopentenyl adenine (2iP). This was the best medium for embryo development and plantlet regeneration. To a certain extent, MS medium containing $1.0 \mathrm{mg} \mathrm{l}^{-1} \mathrm{NAA}+0.6$ $\mathrm{mg} \mathrm{l}^{-1}$ 2iP also produced well developed somatic embryos. The use of $3 \%$ maltose instead of sucrose as a carbon source proved to be good for embryo development.

Of all the $G$. hirsutum cultivars, only Coker 310 FR, SH-131 and LH-900 were embryogenic. However, for SH-131 and LH900 , the response was very slow and most of the embryos were embedded inside leathery callus, which was not normal. Excellent callus for embyrogenesis should be friable. Additionally, the embryos were mostly brown and did not develop beyond globular and heart stage. The embryos were formed on MS medium supplemented with $0.452 \mu \mathrm{M} 2,4-\mathrm{D}$ as well as on medium supplemented with 14.9 $\mu \mathrm{M} 2 \mathrm{iP}+0.537 \mu \mathrm{M}$ NAA.

\section{Levels of reducing sugars}

Reducing sugars are those possessing a free anomeric carbon atom and get oxidized upon transfer of an electron to any oxidizing agent and thus are highly reactive. The endogenous levels of the reducing sugars were determined in all the cultivars under investigation, in order to study their effect on the induction of somatic embryos. It was observed that the level of reducing sugars increases as the period of culture increases in cultivars BD-1, BD-6, Sarvottam, Jawahar Tapti and LH-900 and Cok 312 FR, irrespective of the ploidy level (Figure 2). In case of cultivar SH-131 the level of reducing sugar decreased as the period of culture increased, this may be attributed to the fact that SH-131 is poorly embryogenic. While, in JK-4 the levels of reducing sugars was constant but decreased in case of Khandwa-2, thus indicating that there was no morphogenetic activity (Figure 2).

\section{Levels of total phenol}

Cultivars BD-1, BD-6 and SH-131 showed very high amount of phenols during the initial period of culture (up to 20 days), which decreased at the later stages of development (Figure 3). There was, however, no particular trend in the levels of phenols in the remaining cultivars.

\section{Levels of free amino acids}

Levels of free amino acids (FAA) in callus of different cultivars of cotton at different stages were analysed. It was observed that in case of cultivars BD-1, BD-6, Sarvottam and Jawahar Tapti, Coker 312 FR, it increased up to 40 days and then decreased while in case of SH-131 and LH-900 the levels remained almost constant (Figure 4). Khandwa-2 had higher levels of FAA at 60 days i.e. at the later stages of development. In case of cultivar JK-4 their levels were almost constant or somewhat decreased but the overall level of FAA in JK-4 was almost double of the other cultivars. A general increase in the levels of FAA, which later decreased with time, was observed in the highly embryogenic cultivars, while in the poorly embryogenic and the non-embryogenic cultivars, the levels of FAA remained (Figure 4). 


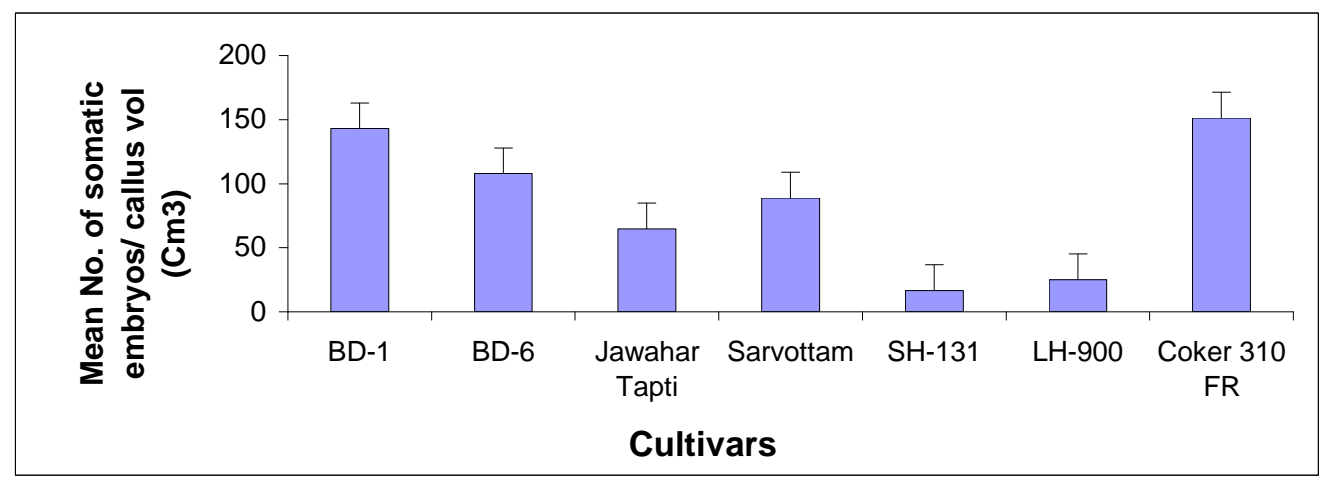

Figure 1: Number of somatic embryos in different cultivars of cotton. Values in the figure are Mean \pm SD of nine determinations (three experiments with three replicates in each case).

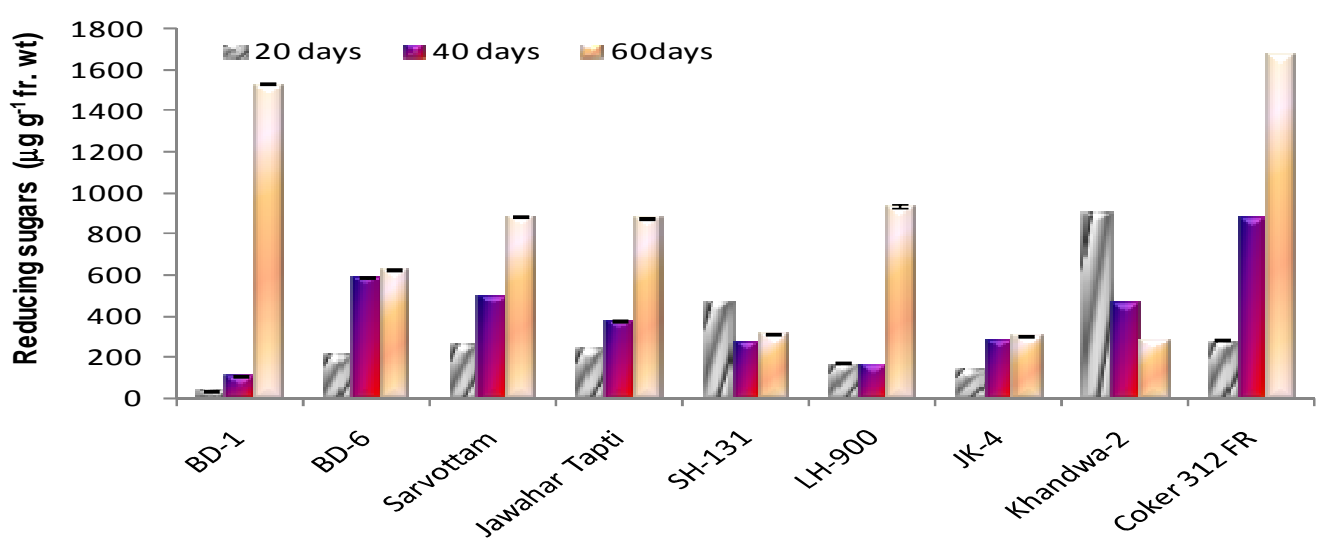

Figure 2: Levels of reducing sugars in different cultivars of cotton at different stages of somatic embryogenesis. Values in the figure are Mean \pm SD of nine determinations (three experiments with three replicates in each case).

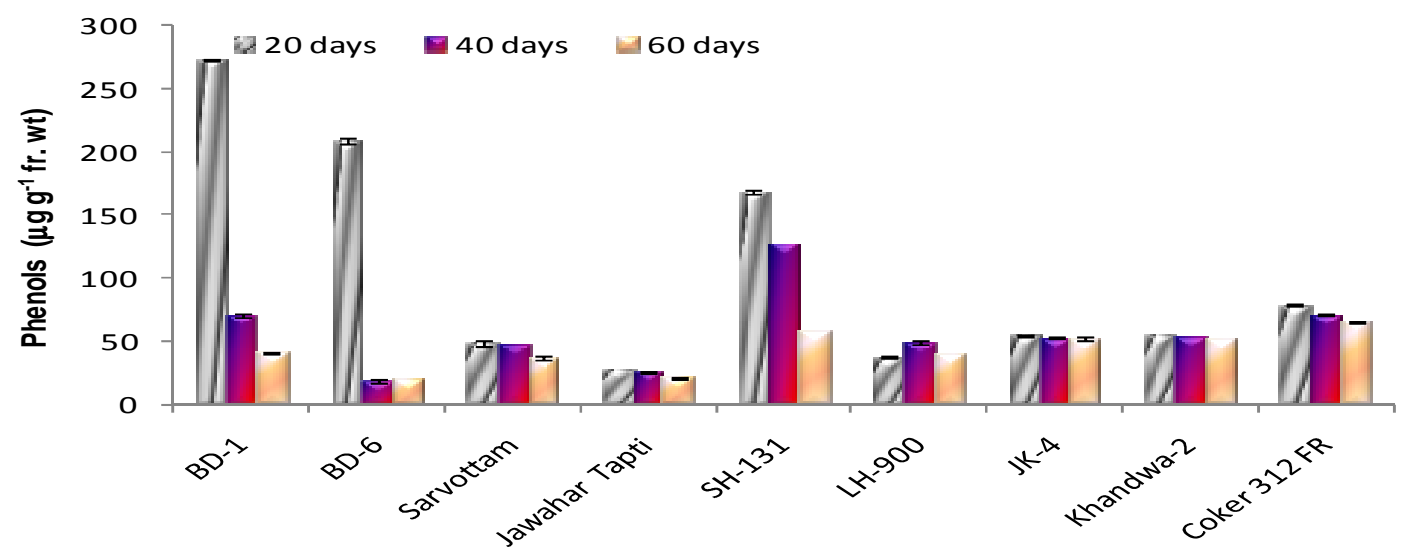

Figure 3: Levels of phenols in different cultivars of cotton at different stages of somatic embryogenesis. Values in the figure are Mean \pm SD of nine determinations (three experiments with three replicates in each case). 


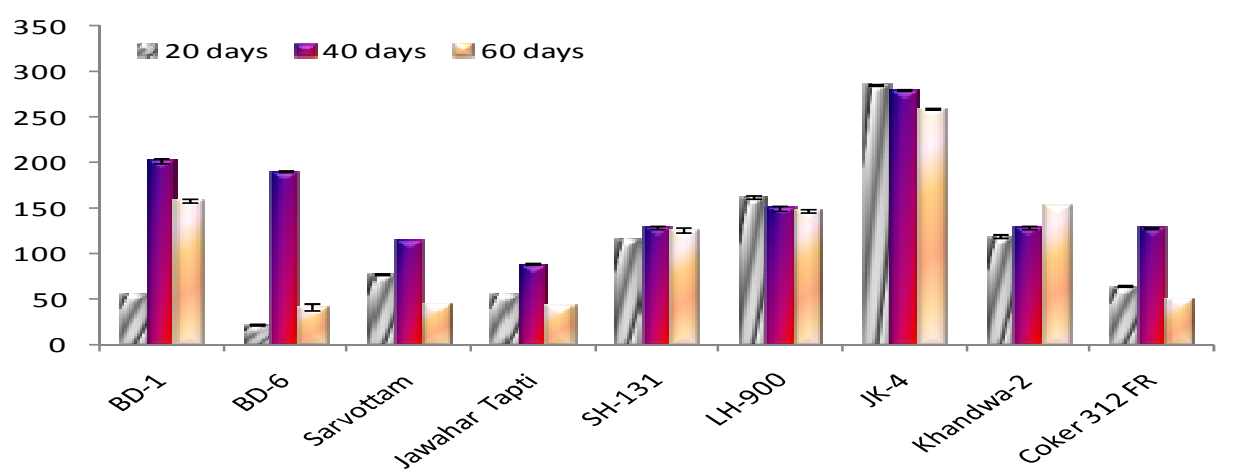

Figure 4: Levels of free amino acids in different cultivars of cotton at different stages of somatic embryogenesis. Values in the figure are Mean \pm SD of nine determinations (three experiments with three replicates in each case).

Table1: Phytohormones used for callus induction, proliferation and embryogenesis on hypocotyl explants of different cultivars of cotton.

\begin{tabular}{|c|c|c|c|}
\hline \multirow[t]{2}{*}{ Cultivars } & \multicolumn{3}{|c|}{ Phytohormone concentration $(\mu \mathrm{M}$} \\
\hline & Callus induction & Proliferation & Embryogenesis \\
\hline BD-1 & \multirow[t]{3}{*}{$10.74 \mathrm{NAA}+4.64 \mathrm{Kin}$} & \multirow{9}{*}{$0.4522,4-\mathrm{D}$} & $0.682,4-\mathrm{D}+2.982-\mathrm{iP}$ \\
\hline BD-6 & & & \\
\hline Sarvottam & & & $5.37 \mathrm{NAA}+1.792-\mathrm{iP}$ \\
\hline SH-131 & \multirow[t]{2}{*}{ 5.37 NAA + 0.464 Kin } & & $0.4522,4-\mathrm{D}$ \\
\hline Vikas & & & No response \\
\hline LH-900 & \multirow{3}{*}{$0.537 \mathrm{NAA}+14.92-\mathrm{iP}$} & & $0.682,4-\mathrm{D}+2.982-\mathrm{iP}$ \\
\hline JK-4 & & & No response \\
\hline Khandwa-2 & & & No response \\
\hline Coker 312 FR & $5.37 \mathrm{NAA}+0.464 \mathrm{Kin}$ & & $0.682,4-\mathrm{D}+2.982-\mathrm{iP}$ \\
\hline
\end{tabular}

2iP: isopentenyl adenine, Kin: kinetin, NAA: Naphthalene acetic acid, 2,4-D: 2,4-dichlorophenoxy acetic acid

\section{DISCUSSION}

All the stages of somatic embryo development viz., globular, heart shaped, torpedo and cotyledonary stages were observed in these cultivars as previously reported by Khan et al. (2006).

In this study, the highest levels of reducing sugars were found in the highly embryogenic cultivars. This is in accordance with earlier observations by Martin et al. (2000), in Medicago, where it was suggested that organogenesis and somatic embryogenesis require energy, which is supplied by the degradation of starch. Carbohydrate metabolism plays an important role in organogenesis as well as in somatic embryogenesis (Martin et al., 2000). Reducing sugars are important in callus formation and differentiation because they are necessary for the formation of reserved cell wall polysaccharides. Similar observation of high levels of reducing sugars have been made for organogenic calli of rice and sweet potato (Mukherjee et al., 2001; Huang and Liu, 2002). Cangahuala-Inocente et al. (2009) recently repoted that the levels of soluble sugars increased during the cotyledonary and heart stages and decreased in torpedo and precotyledonary stages of somatic embryogenesis in Acca sellowiana. These results indicate that reducing sugars can serve as biomarkers for somatic embrogenesis in cotton.

Phenols, including polyphenols are secondary metabolites synthesized from aromatic amino acids including phenylalanine, 
tyrosine and tryptophan. The phenols in plants range from very simple phenolic acids to highly complex compound lignin. Many plant phenols have significant roles possibly in interaction with hormones and also in conferring resistance to insect pests. Cotton plants contain large amount of phenols, which are secreted when the plant is wounded (Khan et al., 2006). However, there are two contrary opinions on the influence of phenolics on plant growth and development. Whereas, some researchers are of the viewpoint that phenolic substances, in tissue culture studies, generally affect in vitro proliferation negatively (Arnaldos et al., 2001), others have reported opposing effects (Lorenzo and Angeles, 2001). In our study, BD-1 and BD-6 produced very high numbers of somatic embryos amongst all the cultivars and thus a very high amount of phenol was observed during the earlier stages of development in the callus of these cultivars. While in other cultivars, very little decrease or almost a constant level was observed. In a similar study, higher concentration of phenols was recorded in non-embryogenic cocoa callus (Alemannoh et al., 2003). Thus, BD-1 and BD-6 could be taken as candidates to study the role of phenols in somatic embryogenesis, even though phenolic content in cotton does not present itself as a clear-cut maker for somatic embryogenesis.

Decrease in the level of phenols at the time of embryo induction is essential for somatic embryogenesis, as high level of phenols is inhibitory, in that they cut off the oxygen supply to the callus, leading to slow growth and ultimately death of the tissue in the non-embryogenic cultivars (Khan et al., 2006). Ozyigit at al. (2007) have also observed a negative correlation between in vitro proliferation and phenol content in cotton ( $G$. hirsutum). Similar observations were reported earlier in Theobroma cacao (Alemannoh et al., 2003). However, in a study by Pinto et al. (2008), the use of anti-oxidants like DTT, PVP and PVPP, which normally prevent phenolic oxidation, was observed to hamper somatic embryogenesis in Eucalyptus globules.

Production of free amino acids is generally believed to be a stress related phenomenon, as such, it is plausible that the increase in free amino acids in our study may have triggered the induction of somatic embryogenesis. The callus of the nonembryogenic cultivars may have died out, due to their inability to overcome the stress posed by the high levels of free amino acids. Similar observations were made in Vigna mungo (Sen et al., 2002) culture, where a rapid increase in the total amount of amino acids during cell proliferation and early stages of somatic embryo formation was observed. Hence, the production of free amino acids may serve as marker for somatic embryogenesis in cotton.

\section{Conclusion}

Embryogenesis is a complex phenomenon especially in cotton, which has proven to be a recalcitrant crop. Though there have been reports of regeneration and transformation in cotton, this is the first report that attempted to understand the factors involved in somatic embryogenesis, the prime step towards regeneration. The study shows that embryogenesis is not only genus- or species-specific but inter-varietal difference also account for the differential biochemical and developmental response. On the whole, all the $G$. arboreum (diploid) cultivars exhibited similar responses in all the experiments but that was not the case with the G.hirsutum (tetraploid) cultivars. Moreover, the data presented in this work show that there are few factors that may serve as markers for somatic embryogenesis in cotton, which need to be empirically determined for any particular cultivar chosen for genetic improvement through embryogenesis.

\section{REFERENCES}

Alemannoh TR, Garga AD, Andary C, Terriere N. 2003. Localization and identification of phenolic compounds in Theobroma cacao L. somatic embryogeneses. An. Bot., 92: 613-623.

Arnaldos TL, Munoz R, Ferrer MA, Calderon AA. 2001. Changes in phenol content during strawberry (Fragaria $x$ ananasa, cv. Chandler) callus culture. Physiol Plant., 113: 315-322.

Cangahuala-Inocente GC, Silveira V, Caprestano CA, Ducroquet JPHJ, Floh 
EIS, Guerra MP. 2009. Dynamics of biochemical and morphophysiological changes during zygotic embryogenesis in Acca sellowiana (Berg.) Burr. Plant Growth Regul., 59: 103-115.

Huang WL, Liu LF. 2002. Carbohydrate metabolisms in rice during callus induction and shoot regeneration induced by osmotic stress. Bot. Bull. Acad. Sin., 43: 107-113.

Iraqi D, Tremblay M. 2001. Analysis of carbohydrate metabolisms enzymes and cellular contents of sugars and proteins during spruce somatic embryogenesis suggests a regulatory role of exogenous sucrose in embryo development. J. Exp. Bot., 52: 2301-2311.

Khan T, Singh AK, Pant RC. 2006. Regeneration via somatic embryogenesis and organogenesis in different cultivars of cotton ( Gossypium SPP.). In Vitro Cell Dev. Biol. Plant, 42: 498-501.

Komamine A 2001. Mechanisms of somatic embryogenesis in carrot suspension cultures. Phytomorphology, 51: 277-288.

Kumria R, Sunnichan VG, Das DK, Gupta SK, Reddy VS, Bhatnagar RK, Leelavathi S. 2003. High frequency somatic embryo production and maturation in to normal plants in cotton (Gossypium hirsutum) though metabolic stress. Plant Cell Rep., 21: 635-639.

Lorenzo JC, Angeles ML. 2001. Sugarcane microporopogation and phenolic excretion. Plant Cell, Tissue Organ Cult., 65: $1-8$.

Martin AB, Cuadrado Y, Guerra H, Gallego P, Hita O, Martin L, Dorado A, Villalbos N. 2000. Differences in the contents of total sugars, reducing sugars, starch and sucrose in embryogeneic and non embryogenic calli from Medicago arborea L. Plant Sci., 154: 143-151.

Merkel SA, Parrot WA, Flinn BS. 1995. Morphogenic aspects of somatic embryogenesis. In In Vitro Embryogenesis in Plants, Thorpe TA (ed). Kluwer Academic Publishers: Dordrecht; 155-203.

Mukherjee A, Debata BK, Mukherjee PS, Malik SH. 2001. Morphohistochemcial characteristics of embryogenic and nonembryogenic callus cultures of sweet potato (Ipomoea batutus L.). Cytobios, 106: 113-24.

Murashige T, Skoog F. 1962. A revised medium for rapid growth and bioassays with tobacco tissue cultures. Physiol Plant., 15: 473-497.

Ozyigit Il, Kahraman MV, Ercan O. 2007. Relation between explant age, total phenols and regeneration response in tissue cultured cotton (Gossypium hirsutum L.). Afri J Biotechnol., 6: 3-8.

Pinto G, Silva S, Park Y-S, Neves L, Araújo C, Santos C. 2008. Factors influencing somatic embryogenesis induction in Eucalyptus globulus Labill.: basal medium and anti-browning agents. Plant Cell, Tissue and Organ Cult., 95: 79-88.

Sadasivam S, Manickam A. 1996. Biochemical Methods. New Age International Publishers: New Delhi.

Sakhanokho HF, Zipf A, Rajasekaran K, Saba S, Sharma GC. 2001. Induction of highly embryogenic calli and plant regeneration in upland (G. hirsutum L.) and Pima ( $G$. barbadense L.) cottons. Crop Sci., 41: 1235-1240.

Sen J, Kalia S, Mukherjee SG. 2002. Levels of endogenous free amino acids during various stages of culture of Vigna mungo (L.) Hepper- Somatic embryogenesis, organogenesis and plant regeneration. Curr Sci., 82: 429-433. 